\title{
Éditorial
}

\section{Plusieurs choses à vous dire mais surtout un grand bravo!}

C'est la seconde fois que je parle d'un livre dans mon éditorial et aujourd'hui c'est avec une joie sans retenue que je vous annonce ma fierté d'accueillir dans les colonnes de Radioprotection, publication de la Société française de radioprotection, un hors-série remarquable « Guide pratique : radionucléides \& de radioprotection » de nos amis D. Delacroix, J.P. Guerre et P. Leblanc.

En effet, la réédition enrichie des précédentes versions paraît aujourd'hui comme numéro spécial du volume 39 de notre revue. C'est pour le comité de rédaction un honneur, une grande joie, et nous remercions les auteurs et le CEA qui les a supportés, de leur confiance pour notre journal, reconnaissance sur un plan national certes et je l'espère sur un plan international, des efforts du comité pour hisser la protection radiologique française au niveau d'excellence qu'elle mérite.

Ce livre est remarquable, il est complet, totalement actualisé, enrichi et... beau. J'ai demandé à Patrick Fracas de nous le présenter dans la rubrique « Revue des livres », quelques pages plus loin...

Le comité 2 de la Commission internationale de protection radiologique publie de nombreux documents concernant les radionucléides mais jamais sous une forme condensée, didactique et facilement utilisable. C'est désormais le cas, avec ce livre, que les auteurs ont longuement élaboré, mettant à jour toutes les données, adaptant aux nouvelles normes les anciennes classifications, prenant également en compte les évolutions de la CIPR, telle la suppression de la LAI. Cet ouvrage, très pratique, permettra de passer d'une réglementation à l'autre, il fait parfaitement le lien entre les documents de la CIPR et les actes de radioprotection quotidiens.

Je suis sûr que ce sera un grand succès, n'hésitez pas à y contribuer. Il doit traîner dans tous les laboratoires et bureaux de toutes les personnes qui travaillent avec des radionucléides.

Seconde chose à vous dire : ce numéro est encore très riche, nous montons en puissance dans le nombre d'articles; continuons, c'est tout ce que l'on peut souhaiter à notre journal.

Troisième point et non des moindres, vous trouverez, dans la rubrique « Lettre à la rédaction », une lettre qui me paraît importante : le point de la Commission européenne sur les aspects éthiques des tests génétiques dans le cadre du travail. Nous vous avions communiqué le site Internet de la commission dans notre rubrique « Internet écl@ire »du numéro précédent (Radioprotection 39(1), 117-125, note @8), Christiane Bardoux de la Commission, que les radiobiologistes connaissent bien, nous a envoyé une lettre claire sur le sujet, je tiens à la remercier, le débat est ouvert.

Henri Métivier

Président du comité de la revue 